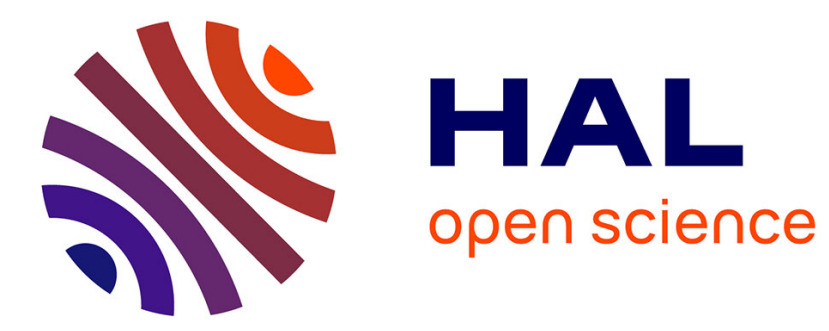

\title{
Image Noise and Digital Image Forensics
}

Thibaut Julliand, Vincent Nozick, Hugues Talbot

\section{To cite this version:}

Thibaut Julliand, Vincent Nozick, Hugues Talbot. Image Noise and Digital Image Forensics. IWDW 2015, Oct 2015, tokyo, Japan. pp.3 - 17, 10.1007/978-3-319-31960-5_1 . hal-01510076

\section{HAL Id: hal-01510076 https://hal.science/hal-01510076}

Submitted on 19 Apr 2017

HAL is a multi-disciplinary open access archive for the deposit and dissemination of scientific research documents, whether they are published or not. The documents may come from teaching and research institutions in France or abroad, or from public or private research centers.
L'archive ouverte pluridisciplinaire HAL, est destinée au dépôt et à la diffusion de documents scientifiques de niveau recherche, publiés ou non, émanant des établissements d'enseignement et de recherche français ou étrangers, des laboratoires publics ou privés. 


\title{
Image Noise and Digital Image Forensics
}

\author{
Thibaut Julliand ${ }^{1}$, Vincent Nozick ${ }^{2}$, and Hugues Talbot ${ }^{1}$ \\ 1 Laboratoire d'Informatique Gaspard-Monge, Equipe A3SI, \\ UMR 8049, Université Paris-Est ESIEE, France \\ thibault.julliand@esiee.fr hugues.talbot@esiee.fr \\ 2 Laboratoire d'Informatique Gaspard-Monge, Equipe A3SI, \\ UMR 8049, Université Paris-Est Marne-la-Vallée, France \\ vincent.nozick@u-pem.fr
}

\begin{abstract}
Noise is an intrinsic specificity of all forms of imaging, and can be found in various forms in all domains of digital imagery. This paper offers an overall review of digital image noise, from its causes and models to the degradations it suffers along the image acquisition pipeline. We show that by the end of the pipeline, the noise may have widely different characteristics compared to the raw image, and consider the consequences in forensic and counter-forensic imagery.
\end{abstract}

Keywords: noise, digital forensics, camera pipeline

\section{Introduction}

From the film grain of analogue cameras to the sensor noise of digital cameras, image noise has always been a concern in the history of image acquisition. Indeed, noise is an unwanted artefact that appears during the image capture process and can take various forms depending on the camera model and the lighting conditions during the image acquisition. For aesthetic reasons or for computer analysis clues, many studies have been concerned with suppressing this noise from the "original" signal. This interest is still an active research topic, particularly considering the increasing number of smartphones equipped with low quality sensors.

Since the noise problem is far from solved, some digital image forensics methods attempt to take advantage of this alteration of the signal to detect image forgeries. Some methods focus on the detection of local noise inconsistencies when some others attempt to identify the noise fingerprint of digital cameras.

The purpose of this paper is to outline the alterations of noise characteristics through the camera pipeline and the usual post-processing, in order to understand what kind of noise one can expect for image forensics purposes. This study first focuses on digital image noise characterisation and estimation. The next part deals with noise alteration during the image acquisition and processing pipeline. We distinguish the processing inherent to the camera pipeline from the usual post processing available on many software packages. Finally, we present some image forensics methods that fail when the image is corrupted with artificial noise. 


\section{Digital Image Noise}

Noise in digital images can come from various sources. Some are physical, linked to the nature of light and to optical artifacts, and some others are created during the conversion from electrical signal to digital data. As noise degrades the quality of an image, various models have been investigated to modelize the image noise for subsequent reduction or removal, at various steps of the image acquisition process.

\subsection{Noise Sources}

The main sources of noise can be divided into two main categories: the physical noise, linked to physics constraints like the corpuscular nature of light, and the hardware noise, linked to mechanical issues in the camera. Physical noise notably includes dark shot noise and photon shot noise[20]. The dark shot noise is created by electronic fluctuations caused by an accumulation of heat-generated electrons in the sensor. It is related to thermal noise, and can be reduced by cooling down the sensor. The photon shot noise, also called Poisson noise, is the one caused by the corpuscular nature of light: as photons arrive irregularly on the photosites, two adjacent pixels supposed to have a similar value can end up with different photon counts. As the name indicates, the photons follow a Poisson distribution. Its effect decreases proportionally to exposure time.

The hardware noise includes Fixed Pattern Noise (FPN), Photon Response Non Uniformity (PRNU) and quantification noise. PRNU and FPN are caused by imperfections in sensors. For the PRNU, the cause is mainly the inhomogeneity of silicon wafers and light variations in which individual sensor pixels convert light to electrical signals. It is most visible in pictures with a long exposure time and does not follow any particular statistical law. As for the FPN, it is caused by dark currents. Like photon shot noise, FPN tends to be reduced in long exposure images. Both of those effects increase with light intensity. While the FPN can be removed by substracting the dark frame, the PRNU is non-linear and as such is very hard to remove. Quantification noise is caused by the analogic-numeric converter. It is hard to quantify, because the process is non-linear, though there are some accepted models. More advanced analysis can be found in [5, 29].

\subsection{Noise Model}

In the literature, the overall noise produced by a digital sensor is usually considered as a stationary white additive Gaussian noise. In [9], Faraji et al. justify the use of the Gaussian model for a specific interval of light intensity. However, this approach tends to overlook several noise components, even if we consider it in a global perspective. Jezierska et al.[15] present a more robust model which also considers a Poisson component. Both of those models take a high-level approach, trying to offer a simplified overall model. In [14], Irie et al. present another approach, which consists in modelising the noise step by step to get to a final formula. While this approach gives extremely precise results, it requires some 
specific data, such as the gain parameter for digital image enhancement, and thus cannot be used for blind modeling.

In the following parts of this paper, we adopt the Poisson-Gauss model from [15]. This model is applied to all the pixel $s$ of the image with :

$$
I_{s}=\alpha Q_{s}+N_{s}
$$

where $Q_{s}$ is analogous to a Poisson distribution $\mathcal{P}\left(u_{s}\right)$ of the "clean" signal $u_{s}$ and $\alpha \in \mathbb{R}$ is a scaling parameter corresponding to the strength of the Poisson component in the noise. $N_{s}$ is analogous to a Normal distribution $\mathcal{N}\left(c, \sigma^{2}\right)$ with mean $c \in \mathbb{R}$ and standard deviation $\sigma>0$.

\subsection{Noise Estimation and Denoising}

Image denoising is a very active research field in the signal and image processing community. However, most existing denoising methods require noise parameters estimation before denoising. Hence, some noise parameters estimation studies have also been proposed. For accuracy purposes, the following overview presents the methods that can handle both Gaussian and Poisson noise.

Foi et al. [11] present such a method that identifies the Gaussian and the Poisson noise. However, this method is subject to an homogeneous image region search that discards a large part of the pixels of a natural image. Thus it may sometimes fail on small regions of the image where homogeneous parts are too small to be considered. Jezierska et al. [15] distinguish pixels that are more subject to Gaussian or Poisson noise from an iterative Expectation-Maximization process. Nevertheless, this method is extremely slow, and thus is impracticable for regular images. Colom and Buades [4] present a PCA noise decomposition approach which gets fast results and is efficient on post-CFA images.

It is important to note that the main purpose of noise estimators is to get a good noise estimation in order to denoise, but not really to get an accurate noise estimation. Thus, a possible approach for estimating the image noise may consist of first using one of the previously mentioned methods to roughly estimate the image noise, then to denoise the image and then subtracting the result from the original noised image. Among the large variety of denoising methods, the NonLocal Means, proposed by Buades et al. [2], performs well. However our tests show that the method lacks accuracy in highly textured zones. Moreover, this method only denoises the Gaussian noise component. Jezierska et al. [16] follows their previous work [15] and still suffers from time computation issue. Dabov et al. [6] introduce the so-called BM3D algorithm that performs a 3D collaborative filtering. This technique performs high-quality denoising for both homogeneous and textured regions, and denoises both the Gaussian and Poisson components.

In this paper, the noise estimation follows the latter approach and the noise estimation is computed from the provided noise image. The Poisson and Gaussian noise parameters are estimated from the difference image between the denoised image by BM3D and the original noised image. We first divide the pixel luminance range into $n$ equal intervals $\mathcal{I}_{i}, i \in[1, n]$. The pixels of the denoised 
image with intensity in $\mathcal{I}_{i}$ are grouped together to compute a variance $\sigma_{i}$ of these pixels in the noise image and a mean value $m_{i}$ in the denoised image. As specified by [11], the noise that appear in the lower and higher pixel intensities is not reliable. Thus, these pixels are discarded from the noise estimation process. The plot of the variance as a function of the mean gives a line which slope corresponds to the Poisson noise parameters and the $y$-intercept corresponds to the Gaussian noise component. An example of this noise estimation is depicted in Figure 1. The intervals $\mathcal{I}_{i}$ are referred to as pixel groups in the following Figures of the paper. In the case of a pure Gaussian noise, this line would be horizontal.

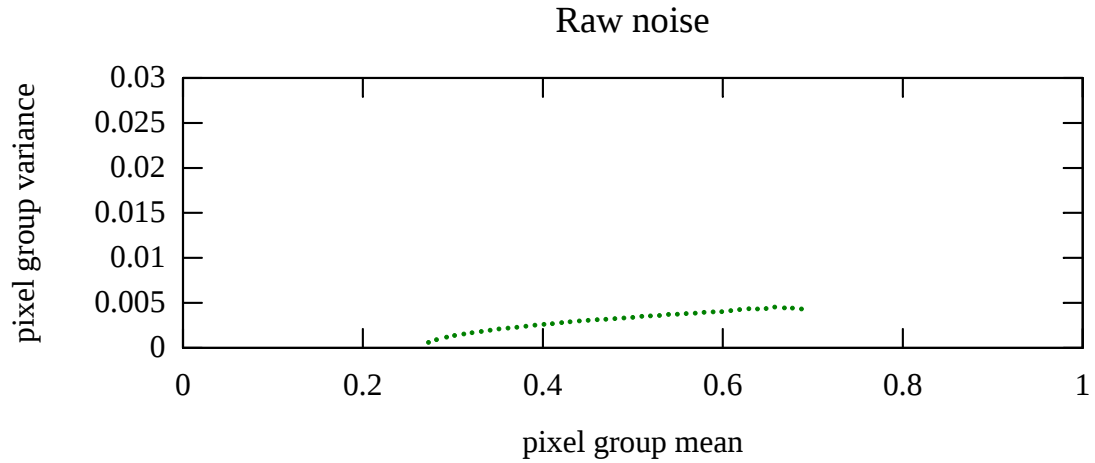

Fig. 1. Pixel group variances according to the group mean. Here, the (first) green channel of the raw image. This image does not use the full intensity range.

\section{Noise Alterations Caused by Image Processing}

\subsection{Noise and Camera Pipeline}

The camera pipeline often differs from one camera to the next, according to the sensor quality and the camera brand. Differences occur according to the processing steps and their ordering, however most pipelines include similar steps. A good description of these steps can be found in [18, chapters 1 and 3]. In this section, we focus on the processings that affect the noise, and show their impact on a raw image. The tests have been implemented using LibRaw [1] on a set of 15 raw images from various cameras. For clarity purposes, we selected one image (Figure 2) of the set with representative results for the figures.

Noise Reduction: It is generally preferable to reduce noise as early as possible in the chain, before signal amplifier operations (notably color correction, gamma correction, edge enhancement and color filter array demosaicking). Some standard denoising methods employ wavelet denoising or Fake Before demosaicking De-noising (FBDD) on each of the four channels (R, G1, B, G2). Figure 3 shows how a light and a full FBDD affect the image noise. 


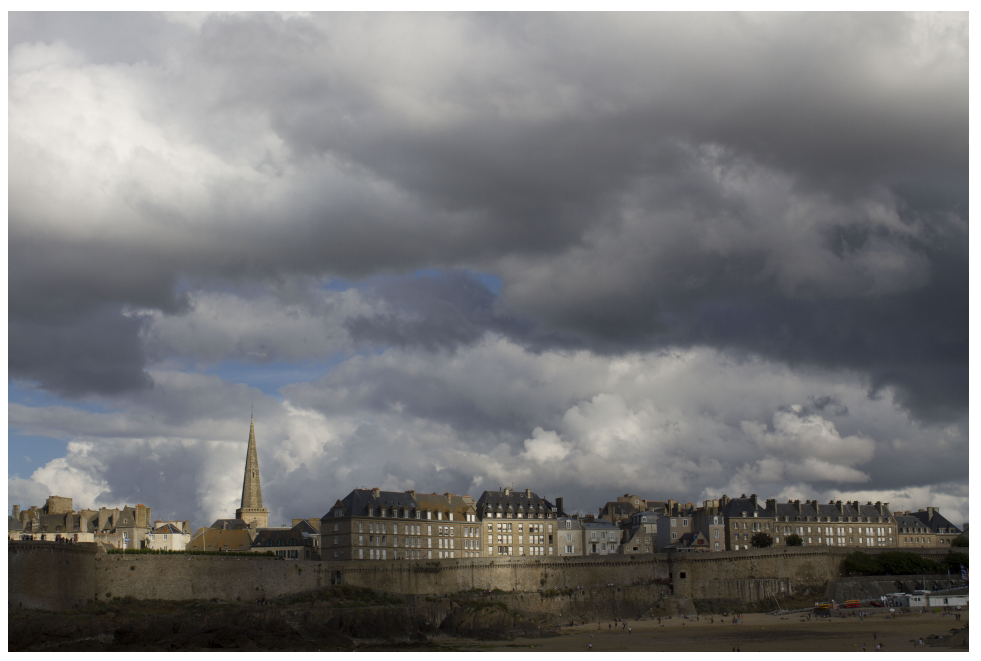

Fig. 2. Input image used for the curves computation. Courtesy of Michel Couprie.

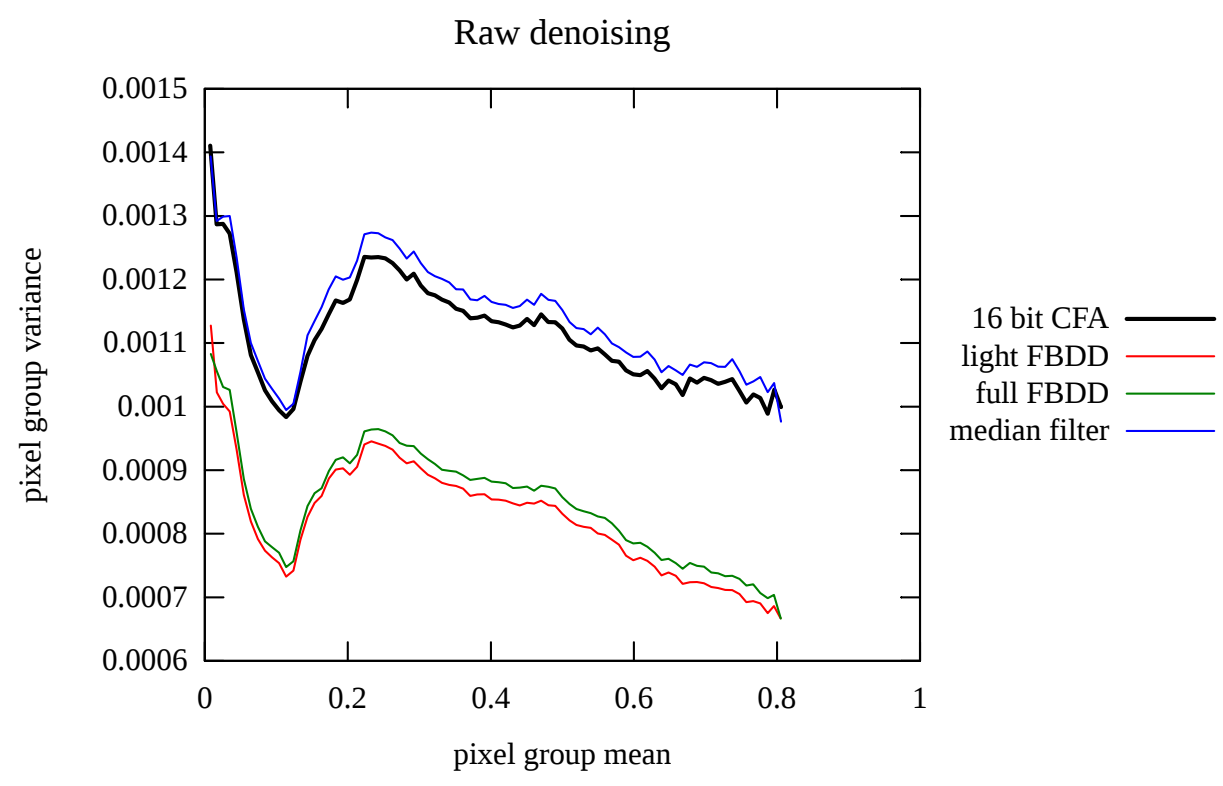

Fig. 3. Comparison of various denoising methods on raw images. For each image, color filter array demosaicking was performed after the denoising step. 
Color Filter Array: The Color Filter Array (CFA) allows a single color to be acquired at each pixel. This means that the camera must interpolate the missing two color values at each pixel. This estimation process is known as demosaicking, and modifies the noise properties that could be found on a raw image. Many demosaicking methods also include edge detection or denoising, like Paliy et al. [22]. Therefore, the CFA can have a strong effect on the noise structure, as shown in Figure 4, using adaptive homogeneity-directed (AHD) interpolation algorithms of dcraw.

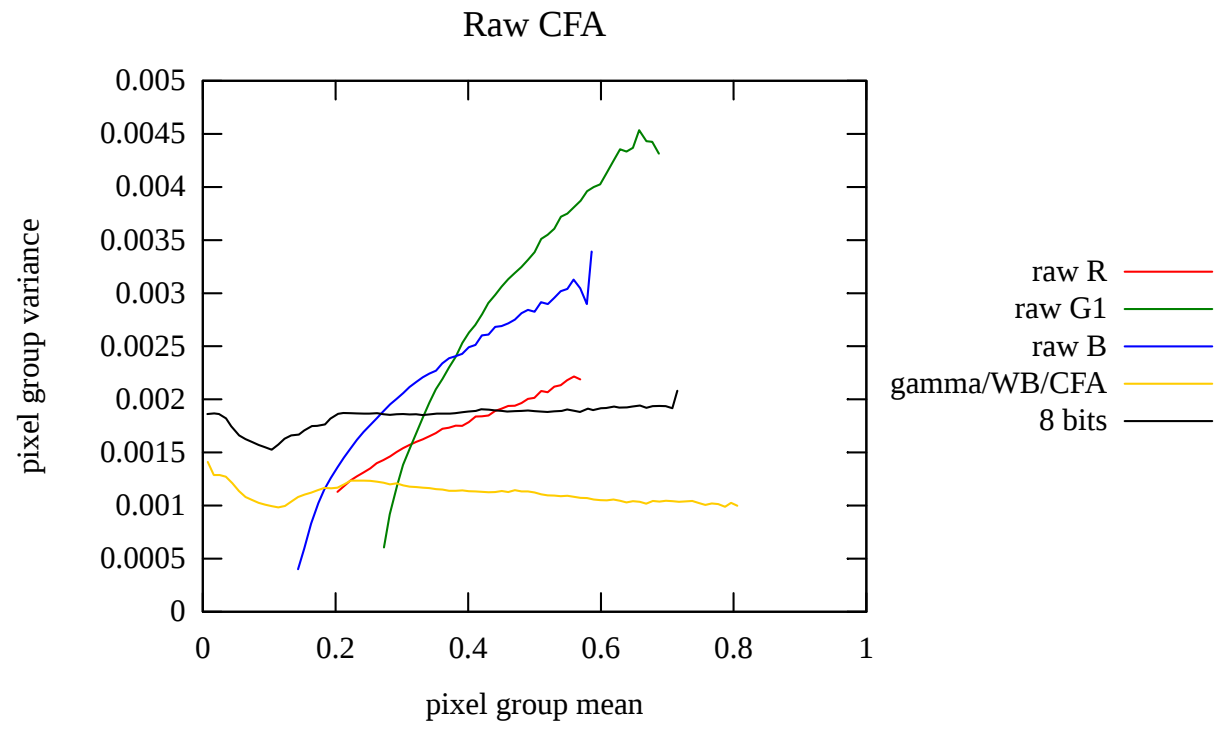

Fig. 4. This Figure depicts the effect of gamma correction, White Balance and CFA demosaicking on the raw channels of the raw image. The combinaison of these 3 steps has a strong effect on the image noise.

White Balance and contrast: White Balance (WB) as well as a contrast operation is just a scaling of all the values of a channel. Since the scaling of a Poisson Gaussian noise remains a Poisson Gaussian noise, the only effect of a WB or a contrast is to enhance or decrease the noise level, however the noise remains present with similar variations.

Bit Depth: The conversion for raw depth, usually from 10 bits to 14 bits, to the 8 bits of the usual image file format is a compression that can have varying effects. Intuitively, we could expect noise levels to be reduced. However, we typically observed that the noise was either at the same level, or even higher, after quantification. There are several possible explanations for this phenomenon: 
first, if the standard deviation is close enough to the conversion quantification step, results can be unpredictable. Second, the quantization actually removes most of the low standard deviation noise, which represents most of the noised pixels, and only leaves the noised pixels with high variations. As a consequence, the calculated standard deviation is much higher, even though the image may look less noisy. This phenomenon can be observed in Fig 4 .

JPEG Compression: JPEG is a lossy compression method with the lossy part predominantly in the high frequencies. Hence, it is not surprising that JPEG compression strongly affect the noise, as depicted in Figure 5. However, our tests show that the global shape of the noise is conserved.

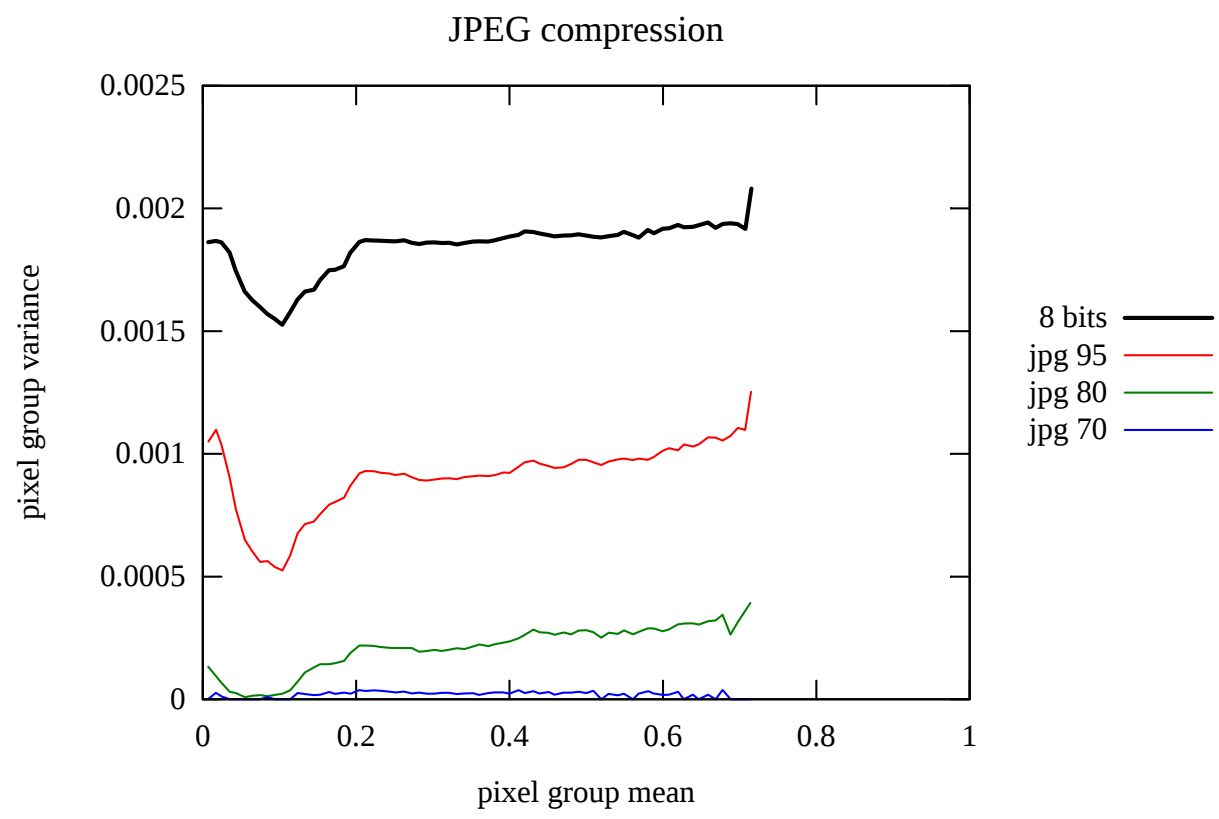

Fig. 5. JPEG compression effect on noise. The bold line is the uncompressed 8 bits image noise and the other curves correspond to the noise after a compression of 95, 80, 70 and 50 .

\section{2 "Legal" Image Enhancements}

This section presents some usual image filters commonly used to enhance the visual image quality. These processes usually do not involve image forgery. The tests were performed on 8 bits digital images extracted from raw images without a denoising process or lossy compression, i.e. they still contain noise. 
Image Interpolation: Image interpolation usually results from image resize, which is one of the most common image processing operation. Image interpolation can also occur for many other reasons, such as image rotation, image perspective transformations (e.g. stereoscopic rectification [21]), radial distortion and chromatic aberration correction, etc. The tests have been conducted with bilinear, bicubic, Lanczo and "area" interpolations for both decimation and zoom. The effect on noise is variable according to the interpolation method. The noise is always decreased but the global noise shape is globally conserved, as shown on Fig. 6.

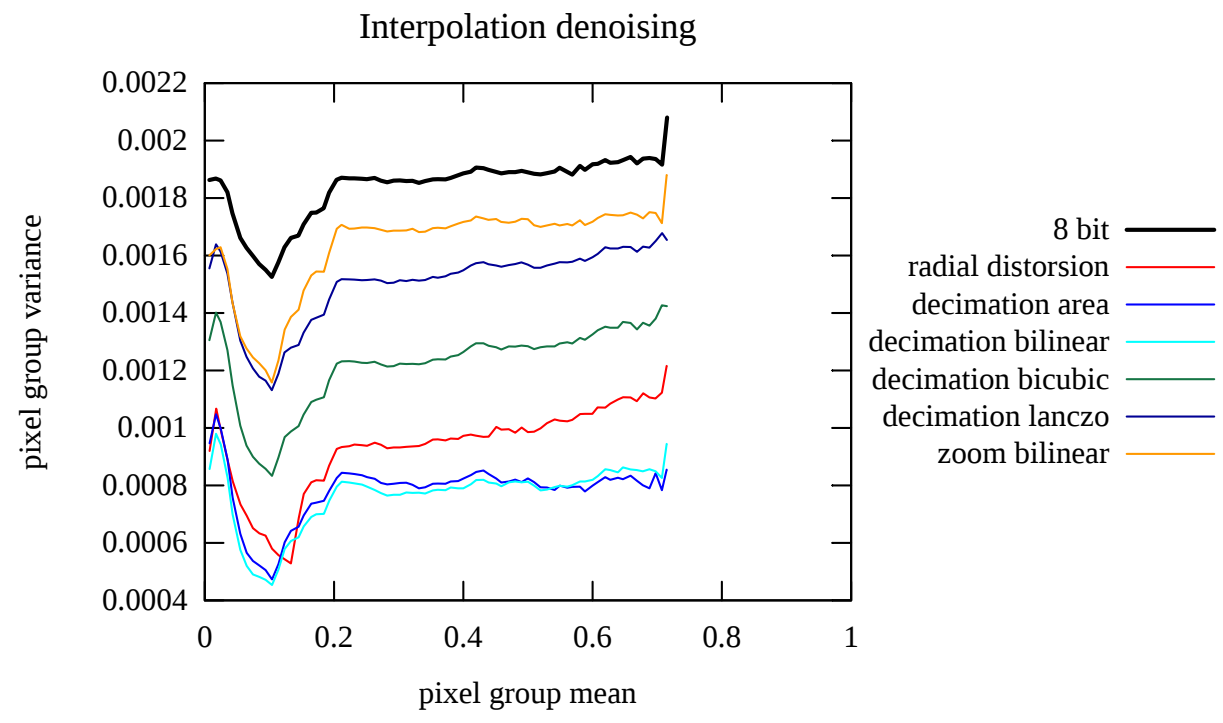

Fig. 6. Interpolation effect on the image noise.

Others: We tested some others images transformations with potential effects on images noise, without significant results. The image saturation process, where colors channels are be mixed together, does not significantly alter the noise. Brightness transformation will just translate the noise to higher pixel intensity levels. Contrast will increase or decrease the noise, but the noise shape remains the same. Image crop will just limit the image surface used for the noise estimation.

\subsection{Noise and Strong Image Forgeries}

This section aims at comparing image noise characteristics after a standard "legal" image enhancement and after a stronger image forgery. The questions are how far the transformed images are from the original in term of noise, and 
if the noise of a strong forgery still makes sens to study. In addition, we denoise the strongly falsified image and renoised it with a light artificial noise.

Figure 7 illustrates one of these experiments with the original image, this image with standard image processing (like non-linear histogram manipulations) and the initial image with strong forgeries. Figure 8 shows that both soft and strong image forgeries significantly impact the noise. More important, this figure demonstrates how an image with artificial noise may exhibit statistics similar to the initial image.

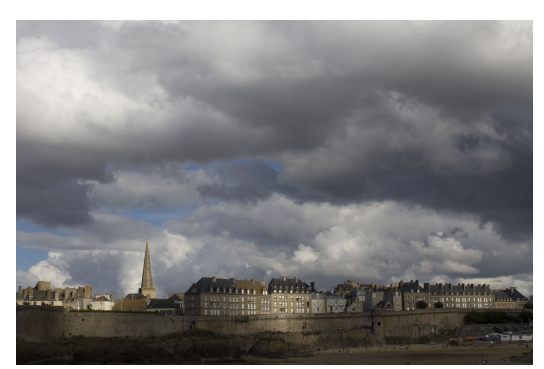

(a) Input image.

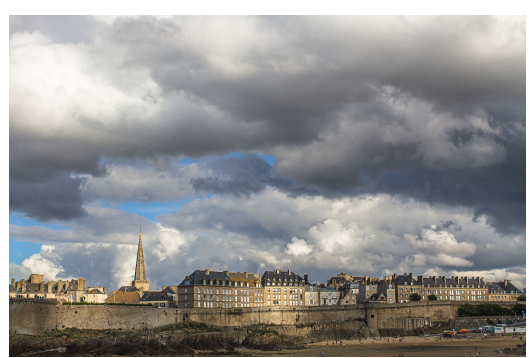

(b) image with "legal" modifications.

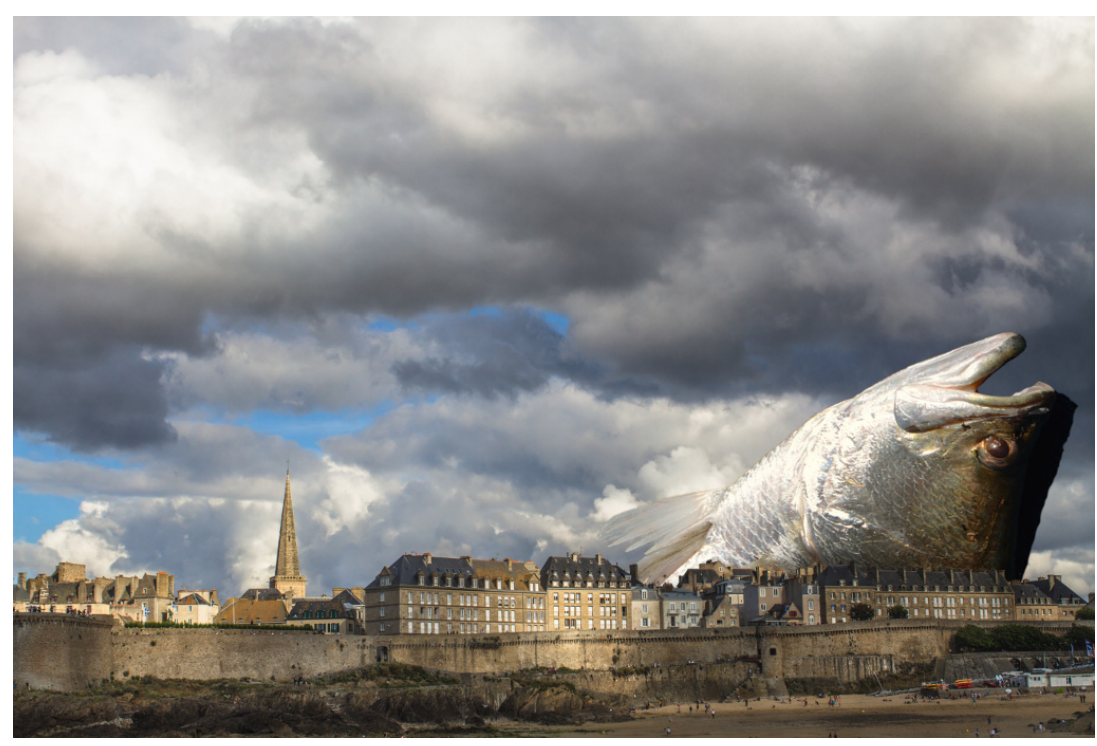

(c) image with strong forgery and virtual noise.

Fig. 7. 7(a): The input image used for the tests. 7(b): The input image with some "legal" transformations such resize, color enhancement, contrast, ... 7(c) Input image with a strong forgery. This image has been denoised and renoised with virtual noise. Courtesy of Michel Couprie and Warren Miconi. 


\section{Image enhancement and strong forgery}

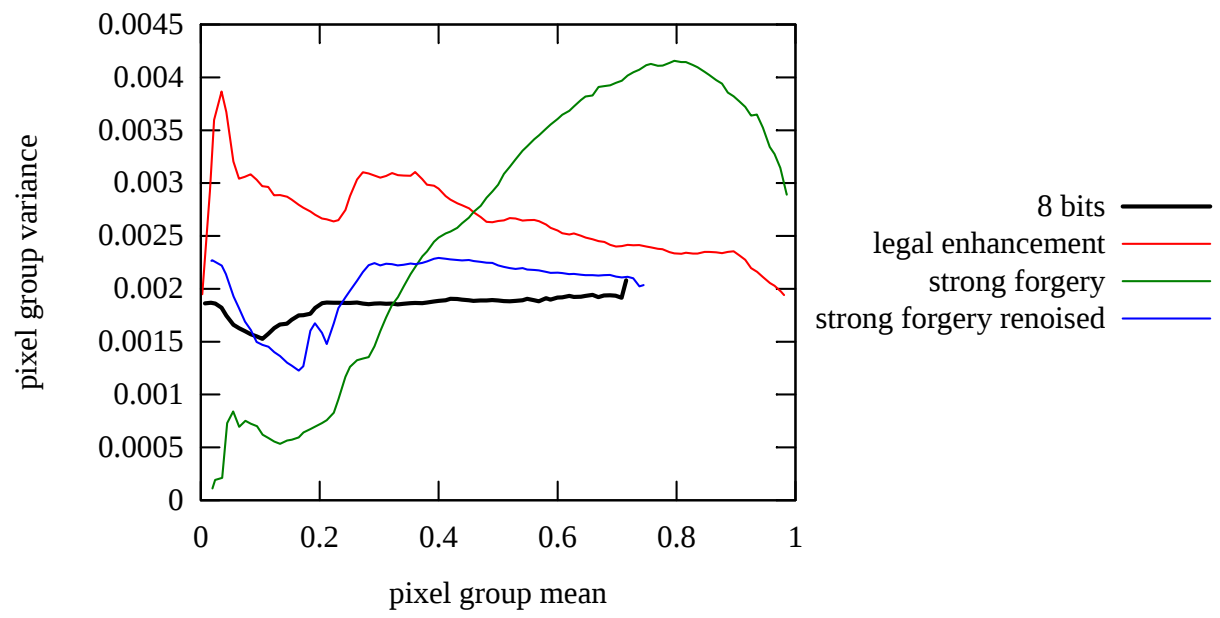

Fig. 8. Image noise curves for "legal" vs. strong image manipulation and for a renoised strong forgery (with an additional contrast modification).

\section{Noise and Forensics Detection}

This section outlines some digital forensic methods based on noise analysis.

Noise Inconsistencies. Mahdian and Saic [19] present a blind method to detect splicing from an image to the other by detecting the noise inconsistencies in the falsified image. The authors first perform a one-level wavelet analysis of the image and then divide the image into a grid to estimate the noise block per block. The authors use white Gaussian noise model. Finally, they merge blocks with similar noise estimation and generate a set of partitions with homogenous noise levels. Pan et al. [23] perform a similar image partition using the kurtosis values of natural images in band-pass filtered domains.

The device sensor fingerprint. The device sensor fingerprint is a sensor pattern noise that can be extracted from the PRNU of a set of images from the same camera. Chen et al. [3], Fridrich [12] and many others like [25, 17] use these fingerprints for device identification. Experiments reported in [3] show promising results even for JPEG compressed images down to a quality factor of 75 .

Computer Graphics. Some image forgeries can include some Computer Graphics (CG) parts when splicing is not possible. These CG image areas may have unusual noise, or no noise. Dehnie et al. [7] look for traces of PRNU in the image and consider the areas with singular PRNU as forgeries. 
Color forgery. Hou et aL. [13] detect hue modification by analysing the correlation of the PRNU from each color channel.

\section{Adding Artificial Noise}

Adding artificial noise can serve several purposes: used on artificial images, it can help to test noise models and algorithms. On natural images, it can be used as a kind of filter, to imprint an image with an old-fashioned grainy feel, or, sometimes, to camouflage an alteration. For our tests, we use the $\mathrm{C}++11$ random number distributions to generate our Poisson and Gaussian distributions.

\subsection{Artificial Images}

Artificial images are ideal to test noise addition models, as they come free of any noise. We have used them to test and confirm the noise model proposed in Eq. (1) for raw images. They also allow to check the consistency of the noise model throughout various intensities and bit depth.

\subsection{Natural Images}

In the case of noise addition in natural images, the questions depend on the objective. If the noise addition is for a purely esthetical value, then the simple addition of a white Gaussian noise is enough. However, if the purpose is to cover other alterations, then a few parallel considerations have to be given thought. First, it is necessary to simulate some FPN, especially if there are several images coming from a single source being altered. Second, the added noise has to be coherent with the type of the image. The type of noise will be different, Gaussian for an 8-bit image and Poisson-Gaussian for a 16 bits one. In this second case, the noised value at pixel $s$ is obtained with $Q$ and $N$ from Eq. (1):

$$
I_{\text {noised }_{s}}=\alpha Q_{s}\left(\frac{1}{\alpha} * I\right)+N_{s}
$$

The last thing to consider is the necessity or not to denoise the image before adding noise. In the case of a splicing, for example, it is necessary to denoise beforehand: indeed, the spliced section may have different noise characteristics than the rest of the image, adding overall noise won't camouflage the difference. A preliminary denoising will help reduce this discrepancy.

\section{Noise and Anti-Forensics}

The objective of this section is to point out some forensics methods that fails to detect digital image forgeries if some artificial noise is added on the falsified images. Indeed, adding artificial noise may affect forgery detection method dealing with noise (Section 4) but also some methods where the pixel values distribution are important. On the following tests, artificial noise is added following the indications of section 5, with a very light Gaussian noise with $\sigma=2.5$, meaning that about $30 \%$ of the pixels are not modified, due to quantization. 
Double JPEG Compression. The double JPEG detection method introduced by Popescu and Farid [24] is based on an effect of the quantization step of the JPEG compression. Adding some noise on the falsified image will remove the quantization artefact and thus strongly decreases the double JPEG detection rate. Figure 9 shows the Fourier analysis of the first DCT coefficient for an image saved in JPEG, then saved again and finally artificially noised and saved in JPEG. The double JPEG artefact are much reduced, and so considerably more difficult to detect.
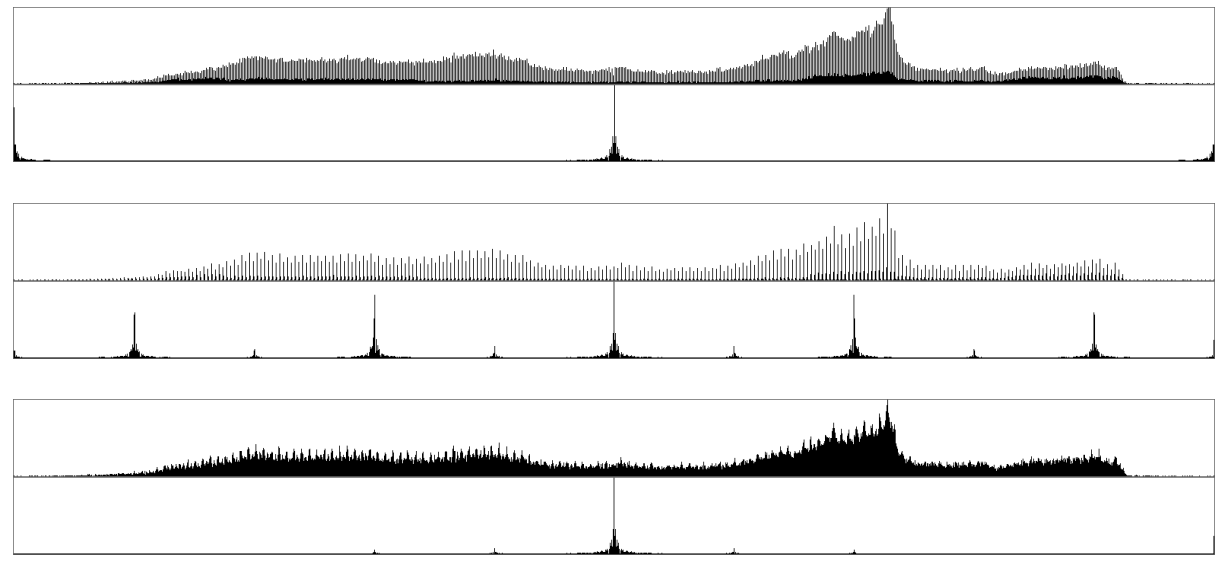

Fig. 9. These graphics correspond to single JPEG (top), double JPEG (middle), and double JPEG + artificial noise + JPEG (bottom). For each image, the upper part is the histogram of the first DCT coefficient and the lower part to its Fourier transform, where the peaks reveal a double JPEG.

JPEG Ghost. The jpeg Ghost method presented by Farid [10] is an extension of the double jpeg that handle local properties of the image. Surprisingly, the method is not altered by noise, unless it is implausibly high. Figure 10 shows the result of this method on a random image with the middle part previously saved in another jpeg quality than the overall image. Note that Stamm et al. [27] successfully disguise the JPEG compression history of an image by adding noise directly on its JPEG DCT coefficients. However, this process leaves slight image alterations that can be detected by [28]. Some recent methods can overcome this alterations issue $[8]$.

Histogram based methods. Some histogram-based methods are used to detect global pixel intensity modifications, typically from Lookup-tables (LUT). Stamm et al. [26] detects the residual peaks of the image histogram resulting from such transformation, by analysing the frequency spectrum of the histogram. 

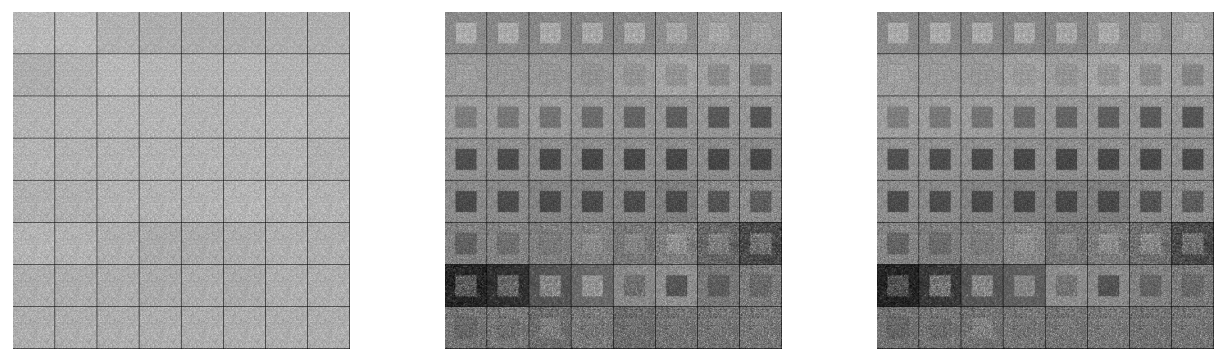

Fig. 10. JPEG ghost [10]: (Left) 64 levels on a random image. (Middle) the 64 levels on the random image where the middle square part is previously saved in another jpeg quality than the overall image. (Right) Same as middle, but with artificial noise before the last JPEG saving.

Adding some noise on the modified image will strongly affect the LUT modification, as depicted in Figure 11.
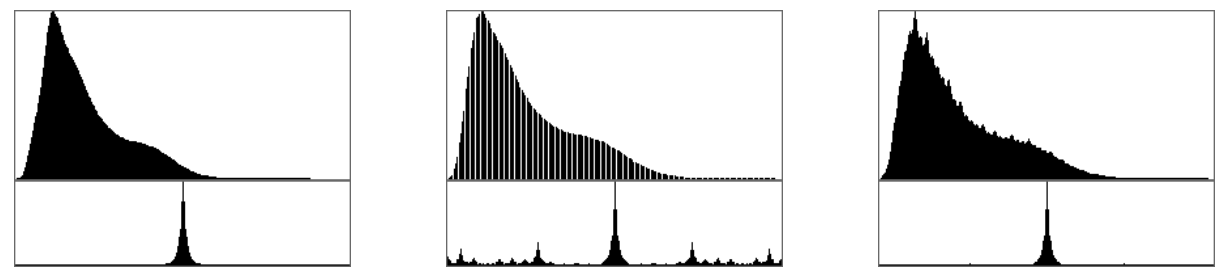

Fig. 11. These graphics correspond to an original image (left), a modified image by applying a LUT, here contrast and brightness (middle), and the middle image with artificial noise (right). For each graphic, the upper part is the histogram of green component of the image and the lower part to its Fourier transform, where the peaks reveal the LUT operation.

\section{Conclusion}

In this article, we have detailed the various sources and models of noise in digital images. Then we have explored a large panel of noise alterations, related to both the acquisition pipeline and the post-processing. We have shown how these alterations affect the quality and intensity of the noise, and study the precise impact of each of those alterations. A major observation we have made is that, by the time we get to a JPEG image, even a high-quality one, the noise is extremely different from its original form in the raw image and is strongly affected by the successive image processing operations. From a statistics point of view, it seems extremely challenging to use this final noise for forgeries detection. Finally, we have looked at the consequences for image forensics and anti-forensics based on noise analysis. 


\section{References}

1. LibRaw 0.17. Image decoder library, 2015.

2. A. Buades, B. Coll, and J.-M. Morel. Non-local means denoising. Image Processing On Line, 2011.

3. Mo Chen, Jessica Fridrich, Miroslav Goljan, and Jan Lukáš. Determining image origin and integrity using sensor noise. Information Forensics and Security, IEEE Transactions on, 3(1):74-90, 2008.

4. M. Colom and A. Buades. Analysis and extension of the PCA method, estimating a noise curve from a single image. Image Processing On Line, 2014.

5. Roberto Costantini and Sabine Susstrunk. Virtual sensor design. Sensors and Camera Systems for Scientific, Industrial, and Digital Photography Applications $V, 2004$.

6. K. Dabov, A. Foi, V. Katkovnik, and K. Egiazarian. Image denoising by sparse 3d transform-domain collaborative filtering. IEEE Trans. Image Process., 16(8):20802095, August 2007.

7. S. Dehnie, T. Sencar, and N. Memon. Digital image forensics for identifying computer generated and digital camera images. In Image Processing, 2006 IEEE International Conference on, pages 2313-2316. IEEE, 2006.

8. Wei Fan, Kai Wang, François Cayre, and Zhang Xiong. A variational approach to JPEG anti-forensics. In Acoustics, Speech and Signal Processing (ICASSP), 2013 IEEE International Conference on, pages 3058-3062. IEEE, 2013.

9. H. Faraji and WJ. MacLean. CCD noise removal in digital images. Image Processing, IEEE Transactions, 2006.

10. Hany Farid. Exposing digital forgeries from JPEG ghosts. Information Forensics and Security, IEEE Transactions on, 4(1):154-160, March 2009.

11. A. Foi, M. Trimeche, V. Katkovnik, and K. Egiazarian. Practical PoissonianGaussian noise modeling and fitting for single image raw-data. IEEE Trans. Image Process., 17(10):1737-1754, 2008.

12. Jessica Fridrich. Digital image forensics using sensor noise. Signal Processing Magazine, 26(2):26-37, 2009.

13. Jong-Uk Hou, Han-Ul Jang, and Heung-Kyu Lee. Hue modification estimation using sensor pattern noise. In Image Processing (ICIP), 2014 IEEE International Conference on, pages 5287-5291. IEEE, 2014.

14. K. Irie, A.E. McKinnon, K. Unsworth, and I.M. Woodhead. A model for measurement of noise in CCD digital-video cameras. Measurement Science and Technology, 2008.

15. A. Jezierska, C. Chaux, J.-C. Pesquet, and H. Talbot. An EM approach for PoissonGaussian noise modeling. In EUSIPCO, pages 2244-2248, August 2011.

16. Anna Jezierska, Emilie Chouzenoux, Jean-Christophe Pesquet, and Hugues Talbot. A primal-dual proximal splitting approach for restoring data corrupted with Poisson-Gaussian noise. IEEE International Conference on Acoustics, Speech and Signal Processing (ICASSP 2012), March 2012.

17. Ashref Lawgaly, Fouad Khelifi, and Ahmed Bouridane. Weighted averaging-based sensor pattern noise estimation for source camera identification. In IEEE International Conference on Image Processing (ICIP 2014), pages 5357-5361, October 2014.

18. Rastislav Lukac. Single-sensor imaging: methods and applications for digital cameras. CRC Press, 2008. 
19. B. Mahdian and S. Saic. Using noise inconsistencies for blind image forensics. Image and Vision Computing, 2009.

20. J. Medkeff. Using image calibration to reduce digital noise in images. 2004 .

21. Vincent Nozick. Camera array image rectification and calibration for stereoscopic and autostereoscopic displays. Annals of telecommunications, 68(11):581-596, July 2013.

22. D. Paliy, V. Katkovnik, R. Bilcu, S. Alenius, and K. Egiazarian. Spatially adaptive color filter array interpolation for noiseless and noisy data. Int. J. Imaging Syst. Technol., 17:105-122, 2007.

23. Xunyu Pan, Xing Zhang, and Siwei Lyu. Exposing image splicing with inconsistent local noise variances. In International Conference on Computation Photography (ICCP), pages 1-10, April 2012.

24. Alin C Popescu and Hany Farid. Statistical tools for digital forensics. In Information Hiding, pages 128-147. Springer, 2005.

25. Kurt Rosenfeld and Husrev T Sencar. A study of the robustness of PRNU-based camera identification. In ISEST/SPIE Electronic Imaging, pages 72540M-72540M. International Society for Optics and Photonics, 2009.

26. Matthew Stamm and KJ Ray Liu. Blind forensics of contrast enhancement in digital images. In Image Processing, 2008. ICIP 2008. 15th IEEE International Conference on, pages 3112-3115. IEEE, 2008.

27. Matthew Christopher Stamm, Steven K Tjoa, W Sabrina Lin, and KJ Ray Liu. Anti-forensics of JPEG compression. In Acoustics Speech and Signal Processing (ICASSP), 2010 IEEE International Conference on, pages 1694-1697. IEEE, 2010.

28. Giuseppe Valenzise, Vitaliano Nobile, Marco Tagliasacchi, and Stefano Tubaro. Countering JPEG anti-forensics. In Image Processing (ICIP), 2011 18th IEEE International Conference on, pages 1949-1952. IEEE, 2011.

29. Saeed V. Vaseghi. Advanced Digital Signal Processing And Noise Reduction. John Wiley \& Sons, 2008. 